\title{
Inserción laboral del enfermero y su desarrollo en ámbito profesional.
}

\section{Nursing job placement and professional development}

Carmen Guaranguay, MSc. ${ }^{(1)}$

Lilia Sánchez, MSc. ${ }^{2}$

(1) Universidad Laica Eloy Alfaro de Manabi, Facultad de Enfermería, Ecuador,carmenguch@gmail.com

(2) Universidad Laica Eloy Alfaro de Manabi, Facultad de Enfermería, Ecuador,lilima28@hotmail.

Contacto: carmenguch@gmail.com

\section{Receptado: 19/02/2018 Aceptado: 16/04/2018}

\section{Resumen}

La inserción laboral es un proceso encaminado a la obtención de un empleo o sea la incorporación activa a un trabajo mientras que el desarrollo profesional es el fruto de la carrera, comprende los aspectos que enriquecen a la persona con vista hacia logro de metas de la organización. El presente estudio tuvo como objetivo analizar las características de inserción laboral, y desarrollo profesional de los licenciados de enfermería de la ULEAM, que posibilite una propuesta de educación continua profesionalizante. La población objeto de estudio la conformaron 116 graduados de las instituciones de salud públicas y privadas de la provincia de Manabi, fue una investigación cuantitativa, descriptiva, retrospectiva de cohorte transversal con un muestreo aleatorio, instrumentos valorados mediante escala de Likert, el análisis se hizo a través de un programa estadístico informático (SPSS).En la investigación se demostró que los graduados están inmersos más en el cuidado directo; se encuentran satisfechos en su trabajo a pesar de que no tienen autonomía en la profesión por falta de especialidades en el campo laboral; los datos aportados al tratado señalan que se debe identificarse, estrategias de acercamiento entre la institución de educación superior y las instituciones empleadoras en el ámbito de empleo y formación continua profesionalizante para mejorar la calidad de atención de salud. 
Palabras claves: Profesional enfermería; ocupación; capacitación; inserción; empleo.

\begin{abstract}
The labor insertion is a process aimed at obtaining a job ie the active incorporation to a job while the professional development is the fruit of the career; it comprises the aspects that enrich the person with a view to achieving the goals of the organization. The present study had as objective to analyze the characteristics of labor insertion, and professional development of the graduates of nursing of the ULEAM, that makes possible a proposal of continuing professional education. The study population was composed of 116 graduates of the public and private health institutions of the province of Manabi, was a quantitative, descriptive, retrospective crosssectional study with a random sampling, instruments valued using Likert scale, the analysis was made Through a computer statistical program (SPSS). In the research it was shown that the graduates are immersed more in direct care; They are satisfied in their work although they do not have autonomy in the profession due to lack of specialties in the labor field; The data provided to the treaty indicate that strategies for the rapprochement between the institution of higher education and the employers' institutions in the field of employment and continuing vocational training must be identified in order to improve the quality of health care.
\end{abstract}

Key words: Professional nursing; occupation; training; insertion.

\title{
Introducción.
}

La inserción laboral se entiende formalmente al conjunto de actuaciones orientadas al conocimiento de la situación laboral de los titulados, y la misma es fundamental para que las personas puedan tener acceso a los medios económicos que le permita mantenerse tanto el cómo su familia (Alcala, 2016), otros autores también definen la inserción laboral como un elemento importante en el análisis de la enseñanzas como también parte de la visión comparada de la opinión de los graduados universitarios (Tokman, Insercion Laboral, Mercados de Trabajo y proteccion social, 2006)

Sin embargo otro autor lo define como un proceso encaminado a la obtención de un empleo y este con lleva a la búsqueda activa del mismo para este fin es necesario tener en cuenta las capacidades y destrezas y formación del sujeto; Comprende la cobertura de las necesidades 
básicas, la formación y la búsqueda y conquista de un trabajo, el mantenimiento del mismo, y la incorporación a otro tipo de actividades, ya sean sociales, culturales, entre otras. (Amaya, 2013)

Por otro lado (Rodriguez, 2000) afirma que los logros educativos no parecen ser muy significativos en la explicación del vínculo laboral que parece responder más a características de los puestos como ocupación, actividad, tamaño de la empresa entre otros; que en relación entre los recursos formativos y las trayectorias laborales."...Por tanto, la educación debería ajustarse a los distintos estados de formación en el que se encuentren los individuos de una sociedad".

Como también la inserción laboral y productiva es el pilar que debe responder a la necesidad de contar con un mercado laboral lo suficientemente flexible para competir en mercados abiertos e inestables. Ello contribuirá a alcanzar un mayor crecimiento del empleo y una reducción del desempleo al reducir la duración de los períodos de ajuste. (Tokman, Inserción laboral,mercados de trabajo y protección social, 2006)

“...La asociación al campo laboral empieza incluso antes de finalizar los estudios a través de las becas pre profesionales, el año de servicio obligatorio, siendo esta una inserción laboral temprana, gracias a las políticas de salud y políticas públicas de empleo del país, que acorto plazo esta experiencia profesional sirve para insertarse en el campo laboral acorde a su profesión".

Según estudio realizado en Cuba, el desarrollo profesional describe las actividades que realiza el enfermero en el ámbito laboral se percibe la capacidad su relación con los créditos que otorga para medir la capacidad a partir de una reviso (Hidalgo, Hidalgo, Garcia, \& Madrazo, Desarrollo Profesional en enfermería, 2002)

Según el sistema cubano de educación refiere que el desarrollo profesional de enfermería depende de cada profesional (Hidalgo, Hidalgo, Garcia, \& Madrazo, Desarrollo Profesional en enfermería, 2002), el cual debe estar en relación al sistema de salud del país; por otra parte también refiere que es responsabilidad del centro de posgrados de la educación superior; que a su vez le permite insertarse en el campo laboral.

Otro relaciona que la formación académica del profesional de enfermería desde el inicio depende los espacios les garantice una formación de especialidad para los que las instituciones 
formadoras deberían evaluar a los que relaciona la demanda en los espacios laborales que también se mide la satisfacción laboral (Hidalgo, Hidalgo, Garcia, \& Madrazo, Desarrollo Profesional en enfermería, 2002) (Barbera M S. A., 2015).

En Brasil destaca que la formación del profesional del enfermero considera su accionar está dirigido a la educación y salud lo que confirma un compromiso social en su esfera de actuación, señala que hay una división del trabajo en relación al cuidado de salud por las limitaciones en el sistema de salud causadas por divisiones sociales en la que se ve afectado el modelo de salud (Chrizostimo, 2015)

La calidad asistencial de enfermería lo considera un proceso eficiente e íntegro donde es importante identificar cuáles son los factores que intervienen para que se implementen acciones que esto permite que se valorare cómo influyen los sistemas de salud en los cuidados que el profesional de enfermería además de que como es la intervención para especificar mecanismo de estrategias de acción (Gabriel1, 2011)

En este sentido el desempeño profesional se manifiesta como actividad práctica social del Profesional de enfermería, que en su relación con su realidad asistencial, no solo logran el conocimiento de esa realidad sino su transformación y la trasformación de sí mismos que define de acuerdo a sus conocimientos adquiridos pero que también son evaluadas. (Aguilar, Lamadrid, \& Saavedra, mar. 2016)

Otro estudio relacional al desempeño del personal de enfermería en ámbito de su labor profesional en tanto a su capacitación y las formas de actuar en el escenario laboral mismo que le permite demostrar sus conocimientos habilidades que se ven reflejadas al definir su profesionalismo en el campo laboral que le permita ser visibilizado (Carmen L. Balseiro Almario, 2012)

La satisfacción de los profesionales esta en relación a su entorno con lo que se realiza surgen otros campos laborales que también responden a las exigencias de este siglo que les permitirá adaptarse en los campos laborales en relación al trabajo que desempeñan. (Solano-Aguilar2, 2010)

La satisfacción de los profesionales esta en relación a su entorno con lo que se realiza surgen otros Campos laborales que también responden a las exigencias de este siglo que les permitirá 
adaptarse en Los campos laborales en relación al trabajo que desempeñan. (Solano-Aguilar2, 2010)

En el estudio realizado en Murcia refiere que la eminente necesidad en los servicios de salud repercute de forma directa en los profesionales de en el desempeño profesional enfermería y al respecto de su Formación que se debería tomar en cuenta los grados académicos como especialidad diplomados y que esta sea de forma extendida que además debe haber respuesta a sus capacidades y que las organizaciones generen propuesta al modelo además de la formación continuada desarrollada (Barbera M C. A., 2015) .

El estudio mediante revisiones literarias acerca del trabajo del profesional de enfermería, destaca que el ambiente laboral influye en las actividades demandadas y que además provocan efectos, como son las alteraciones en su estado físico y psíquicos y que las instituciones deberán tomar en cuenta que estas condiciones favorezca ambiente de trabajo acorde para que la calidad de atención repercuta tanto para el usuario como la del profesional que está prestando su servicio además de conservar sus derechos. (Palma F, 2015)

El propósito de la investigación es proponer una formación profesionalizante como el desarrollo de especialidades y el doctorado lo que permitirá al profesional mantener su status y capacidad de autonomía y mejorar la calidad de atención sanitaria en beneficio de la comunidad.

\section{Materiales y métodos.}

Para recabar la información se aplicó el método empírico como la encuesta cerrada a los licenciados de enfermería en función correspondientes a la cohorte del 2001 al 2011, lo que permito conocer la problemática y el objeto de investigación.

La modalidad de investigación es descriptiva, retrospectivas de cohorte transversal, realizada durante el año 2016, a los profesionales de enfermería, de las instituciones públicas, privadas y ONG; la población fue de 360 enfermeros, según registros de secretaria general, la muestra de estudio estuvo constituida por 116 profesionales, el sistema de selección de la muestra fue aleatoria para su aplicación, con un nivel de confianza del $95 \%$ y un margen de error del 5\%; El instrumento aplicado fue la encuesta con escala Likert, el análisis es cuantitativo y se realizó a través del programa estadístico SPSS. 
Los criterios de inclusión que se tomaron en cuenta fueron los profesionales de enfermería graduados del 2001-2011, que hayan estado laborando en las instituciones públicas, privadas y ONG, graduadas en la Universidad Laica Eloy Alfaro de Manabi y de exclusión los profesionales que estén realizando el año rural, que no estén ejerciendo su profesión en el país, que estén fuera de la cohorte estudiada, licenciadas con funciones de auxiliar de enfermería.

\section{Resultados}

En la investigación realizada mediante encuesta a los licenciados en enfermería de las instituciones de salud públicas y privadas y ONG; en relación a las variables inserción laboral y desarrollo profesional se obtuvo los siguientes resultados.

\begin{tabular}{|c|c|c|c|c|c|c|c|}
\hline & & \multicolumn{5}{|c|}{ Formación profesional } & \multirow[t]{2}{*}{ Total } \\
\hline & & $\begin{array}{l}\text { Enfer } \\
\text { mera }\end{array}$ & $\begin{array}{c}\text { Licenciad } \\
\mathrm{a}\end{array}$ & $\begin{array}{l}\text { Espec } \\
\text { ialista }\end{array}$ & $\begin{array}{l}\text { diplomado } \\
\text { Superior }\end{array}$ & Magíster & \\
\hline \multirow{5}{*}{$\begin{array}{l}\text { tiempo que labora } \\
\text { en la institución }\end{array}$} & $>5$ & 5 & 56 & 0 & 0 & 2 & 63 \\
\hline & $5-10$ & 0 & 29 & 1 & 1 & 1 & 32 \\
\hline & $\begin{array}{l}11- \\
15\end{array}$ & 1 & 9 & 0 & 0 & 1 & 11 \\
\hline & $\begin{array}{l}16- \\
20\end{array}$ & 0 & 2 & 0 & 0 & 0 & 2 \\
\hline & $\begin{array}{l}21 \mathrm{y} \\
\mathrm{mas}\end{array}$ & 0 & 6 & 0 & 0 & 2 & 8 \\
\hline \multicolumn{2}{|l|}{ Total } & 6 & 102 & 1 & 1 & 6 & 116 \\
\hline
\end{tabular}

Tabla 1. Formación profesional-experiencia laboral

Fuente: Enfermeras de las instituciones de salud públicas y privadas

De 116 profesionales encuestados, 102 responden tener grado de licenciatura; 1 especialista, 1 con diplomado superior, 6 con grado de magister y 6 con título de Enfermera. De ellos, 63 tienen menos de cinco años de ejercicio; 32 entre 5-10 años experiencia laboral; 11 profesionales entre 11-15 años de trabajo; 2 licenciadas de 16-20 años de laboral y 8, asumen 21 años y más de desempeño.

\begin{tabular}{|l|l|l|l|l|l|}
\hline \multicolumn{2}{|c|}{} & \multicolumn{2}{|l|}{ Nivel de atención } & \multirow{2}{*}{ Total } \\
\cline { 3 - 7 } & Primaria & Secundaria & Terciaria & \\
\hline Instituciones donde trabaja & MSP & 15 & 28 & 4 & 47 \\
\cline { 2 - 7 } & IESS & 3 & 29 & 2 & 34 \\
\cline { 2 - 6 } & FAE & 0 & 1 & 0 & 1 \\
\hline
\end{tabular}




\begin{tabular}{|l|l|l|l|l|l|}
\hline & privada & 32 & 2 & 0 & 34 \\
\hline Total & & 50 & 60 & 6 & 116 \\
\hline
\end{tabular}

Tabla 2. Nivel de atención- Institución donde labora

Fuente: Enfermeras de las instituciones de salud públicas y privadas

Al preguntar a los licenciados/as de enfermería en que instituciones laboran, 82 profesionales responden que trabajan en instituciones públicas como: Ministerio de salud Pública, Instituto de seguridad Social y 34 laboran en instituciones privadas, de los cuales 50 licenciados/as se desempeñan en el nivel de atención primaria de salud, 60 secundaria y 6 terciaria; Es evidente que las instituciones de salud públicas son las que mayormente acogen a los profesionales.

Al relacionar la categoría de la institución empleadora y la satisfacción del desarrollo profesional del enfermero/era, se verifica que 61, profesionales se encuentran muy satisfechos con su trabajo, 37 satisfechos, y 18 no satisfechos; se puede verificar que los licenciados a pesar de no tener especialidades en el área de desempeño se encuentran muy satisfechos.

\begin{tabular}{|l|l|l|l|l|l|l|}
\hline \multicolumn{2}{|c|}{} & \multicolumn{2}{l}{$\begin{array}{l}\text { Satisfacción del desarrollo profesional del licenciado } \\
\text { enfermería }\end{array}$} & Total \\
\cline { 3 - 7 } & & $\begin{array}{l}\text { muy } \\
\text { satisfecho }\end{array}$ & satisfecho & $\begin{array}{l}\text { Medianame } \\
\text { nte } \\
\text { satisfecho }\end{array}$ & $\begin{array}{l}\text { no } \\
\text { satisfecho }\end{array}$ & \\
\hline $\begin{array}{l}\text { Categoría de la } \\
\text { institución empleadora }\end{array}$ & Acreditada & 30 & 21 & 3 & 8 & 62 \\
\cline { 2 - 7 } & $\begin{array}{l}\text { sin } \\
\text { acreditar }\end{array}$ & 7 & 5 & 0 & 2 & 14 \\
\cline { 2 - 7 } & $\begin{array}{l}\text { por } \\
\text { acreditar }\end{array}$ & 24 & 11 & 0 & 5 & 116 \\
\hline Total & 61 & 37 & 3 & 15 & \\
\hline
\end{tabular}

Tabla $\mathrm{N}^{\circ}$ 3, Satisfacción laboral-categoría de la institución

Fuente: Enfermeras de las instituciones de salud públicas y privadas

\begin{tabular}{|c|c|c|c|c|c|c|}
\hline & & \multicolumn{4}{|c|}{$\begin{array}{l}\text { Recomendaciones para la Facultad de Enfermería de la } \\
\text { ULEAM, en capacitación profesional continua }\end{array}$} & \multirow[t]{2}{*}{ Total } \\
\hline & & Académica & $\begin{array}{l}\text { Innovación } \\
\text { tecnológica }\end{array}$ & Valores & $\begin{array}{l}\text { capacidad de } \\
\text { liderazgo }\end{array}$ & \\
\hline \multirow[t]{3}{*}{$\begin{array}{l}\text { expectativa } \\
\text { profesional }\end{array}$} & $\begin{array}{l}\text { Elevar el } \\
\text { nivel } \\
\text { profesional }\end{array}$ & 58 & 17 & 9 & 11 & 95 \\
\hline & asensos & 3 & 5 & 0 & 1 & 9 \\
\hline & $\begin{array}{l}\text { Mejora } \\
\text { salarial }\end{array}$ & 6 & 3 & 2 & 1 & 12 \\
\hline \multicolumn{2}{|l|}{ Total } & 67 & 25 & 11 & 13 & 116 \\
\hline
\end{tabular}


Tabla $N^{\circ}$, Recomendaciones en relación a la capacitación continua de los profesionales

Fuente: Enfermeras de las instituciones de salud públicas y privadas

En relación a las recomendaciones que hacen los/las licenciados en enfermaría a la Institución de educación superior de los 116, encuestados 67, opinan promover el nivel académico, 25, innovación tecnológica, 11, valores, 13, capacidad de liderazgo; paralelamente 95, de ellos refieren que la capacitación continua les permite elevar el nivel profesional, 9 asensos, 12 mejora salarial; ya que esto les permite participar en puestos estratégicos de liderazgo.

Las Licenciadas/dos, refirieron que una vez titulado la Universidad les oferto capacitación profesional continua. 16, afirman que siempre, 6, algunas veces, 94, ninguna; y en relación al empleo se evidencio que a 16 profesionales se les oferto trabajo, 7 algunas veces, 93, ninguno; cabe señalar que una vez titulado la universidad no le oferto empleo, así como capacitación profesional continua; por lo tanto es hora de consensuar acuerdos estratégicos entre las Instituciones Educación superior y las Instituciones empleadoras

\begin{tabular}{|l|l|l|l|l|l|}
\hline \multicolumn{2}{|c|}{} & \multicolumn{2}{l|}{$\begin{array}{l}\text { Una vez graduado la universidad Laica Eloy } \\
\text { Alfaro de Manabí le oferto capacitación } \\
\text { profesional continua }\end{array}$} & Total \\
\cline { 3 - 6 } & siempre & $\begin{array}{l}\text { Algunas } \\
\text { veces }\end{array}$ & Ninguna & \\
\hline $\begin{array}{l}\text { Una vez titulado la } \\
\text { universidad le oferto } \\
\text { empleo }\end{array}$ & siempre & 15 & 1 & 0 & 16 \\
\cline { 2 - 6 } & Algunas veces & 1 & 2 & 4 & 7 \\
\cline { 2 - 6 } & Ninguna & 0 & 3 & 90 & 93 \\
\hline Total & 16 & 6 & 94 & 116 \\
\hline
\end{tabular}

Tabla $N^{\circ} 5$, Oferta de capacitación profesional continua - trabajo Fuente: Enfermeras de las instituciones de salud públicas y privadas

\section{Discusión}

En el presente estudio se analizaron las características que adopta la Inserción laboral y el Desarrollo profesional de las egresadas (os) de la Facultad de Enfermería de la ULEAM, a partir del estudio de once generaciones que se graduaron comprenden los años 2001 al 2011.

En relación a la inserción laboral, un alto porcentaje de enfermeros se encuentra laborando en instituciones públicas y privadas en los niveles de atención primaria y secundaria, orientada a la detección y tratamiento de la enfermedad lo que permite identificar el posicionamiento en la asistencia sanitaria accesible al individuo familia y comunidad (Balseiro, 2012). Al realizar el 
estudio en la Universidad Laica Eloy Alfaro de Manabi, se encontró una similitud a los escenarios donde se desempeñan.

El estudio de los profesionales de enfermería en de su desempeño profesional está comprendido entre edades 20 y 40 años, la experiencia laboral de menos de 5 años ocupa un lugar significativo pero que se justifica con las edades tratándose de una población joven, competitiva, productiva, que les permite desempeñarse no solo en un trabajo sino en varios campos de la salud; para las instituciones empleadoras se convierte en una fortaleza de tal modo que la producción será en beneficio de salud de la población atendida, también es visible el mayor número de Licenciados en ejercicio del cuidado directo mas no los espacios administrativos como gerencia o coordinación administrativa.

También se pudo evidenciar que los licenciados en enfermería, en un gran porcentaje están satisfechos con su remuneración, el trabajo, las oportunidades profesionales, los beneficios, por lo tanto es importante la motivación y satisfacción porque contribuye eficiencia y eficacia al logro de los objetivos organizacionales, lo cual implica una mejora en los resultados de la organización.

\section{Conclusiones.}

En este trabajo se ha observado que los licenciados en enfermería se desempeñan más en instituciones del Ministerio de Salud Publicas, en relación a las privadas, con alto porcentaje en áreas curativas que preventivas, de lo que se concluye que el enfoque de la enfermera es más curativo como cuidado directo que preventivo.

En relación al desarrollo profesional y la expectativa en el campo laboral de los/las licenciadas tienen la necesidad de elevar el nivel profesional y la mejora salarial, a través de la educación continua profesionalizante, por que los estudios de especialización, tienen como objetivo profundizar y ampliar los conocimientos y destrezas que requiere el ejercicio profesional en una área específica permitiéndole tomar decisiones actuar de manera independiente, tener seguridad en los procedimientos que realizan y comprometerse con la seguridad de los pacientes.

Finalmente cabe señalar que una vez titulado la universidad no le oferto empleo, así como capacitación profesional continua; por lo tanto hora de consensuar acuerdos estratégicos entre las Instituciones Educación y las Instituciones empleadoras como demanda la Ley de 
Educación Superior en el artículo 11 literal e) de la Ley Orgánica de Educación Superior dice textualmente: "Promover y propiciar políticas públicas que promuevan una oferta académica y profesional acorde a los requerimientos del desarrollo nacional";

Como también Ofrecer el servicio de la Bolsa de empleo creando una base de datos de graduados, para que las instituciones ubicadas principalmente en la zona 4 del país, dispongan de manera oportuna en la página web de la universidad, para los profesionales que necesiten ejercer sus actividades o servicios profesionales.

\section{Referencias}

Aguilar, A. E., Lamadrid, M. d., \& Saavedra, M. O. (marzo de mar. 2016). El desempeño de los profesionales de Enfermería. Rev Cubana Enfermer vol.32 no.1 Ciudad de la Habana ene.-mar. 2016, 32(1).

Alcala, U. d. (17 de Marzo de 2016).

https://portal.uah.es/portal/page/portal/servicio_orientacion/repositorio/archivos/PC _PC07_Insercion.pdf. Obtenido de https://portal.uah.es/portal/page/portal/servicio_orientacion/repositorio/archivos/PC_P C07_Insercion.pdf: https://portal.uah.es

Amaya, T. (24 de Junio de 2013). http://www.dspace.uce.edu.ec/bitstream/25000/2758/1/TUCE-0007-65.pdf. Obtenido de http://www.dspace.uce.edu.ec/bitstream/25000/2758/1/T-UCE-0007-65.pdf: http://www.dspace.uce.edu.ec

Balseiro, C. Z. (2012). Insercion laboral, desarrollo profesional y desempeño institucional del Plan Unico de Espeialización Enfermeria . Enfermeria Universitaria UNAM, 23.

Barbera M, C. A. ( 2015). Formación académica del profesional de enfermería y su adecuación al . Rev. Latino-Am. Enfermagem, 400-406.

Barbera M, S. A. (mayo de 2015). Formacion Academica del profesional de enfermeria y su adecuacion en el trabajo. Latino-Enfermagen. 
Carmen L. Balseiro Almario, M. R. (Enero-Marzo de 2012). Inserción laboral, desarrollo profesional. Enfermería Universitaria ENEO-UNAM •Vol 9. • Año. $9 \cdot$ No. 1 • EneroMarzo 2012 17, 9(1).

Chrizostimo, M. M. (octibre de 2015). La formación profesional del enfermero: 'estado del arte'. Enfermeria global (40).

Gabriel1, C. S. (sep de 2011). Utilización de indicadores de desempeño en servicio de enfermería de. Rev. Latino-Am. Enfermagem.

Hidalgo, G. R., Hidalgo, G. M., Garcia, D. E., \& Madrazo, G. N. (2002). Desarrollo Profesional en enfermería. Cubana de Enfermería.

Hidalgo, G. R., Hidalgo, G. M., Garcia, D. E., \& Madrazo, G. N. (2002). Desarrollo Profesional en enfermería. Cubana de Enfermería.

Palma F, V. S. (2015). EL TRABAJO DEL PROFESIONAL DE ENFERMERÍA:. CIENCIA Y ENFERMERIA XXI, 11-20, .

Rodriguez, M. (2000). Insercion laboral de los jovenes españoles. Alcala: Universidad de Castilla-Mancha.

Solano-Aguilar2, S. (enero -diciembre de 2010). Satisfacción laboral en profesionales de enfermería1. CUIDARTE, $1(1)$.

Tokman, V. (2006). Insercion Laboral, Mercados de Trabajo y proteccion social. En V. Tokman. Santiago de Chile: Cepal.

Tokman, V. (2006). Inserción laboral,mercados de trabajo y protección social. Santiago de Chile: CEPAL,Naciones Unidas. 